\title{
Monitoring Neonatal Peripheral Circulation by Electrocardiogram-to-Oximeter Pulse Velocity
}

\author{
MASAYA OISHI, HIROSHI NISHIDA, KAZUHIKO KABE, AND JUN HOSHI \\ Maternal And Perinatal Center, Tokyo Women's Medical College, Tokyo, Japan
}

\begin{abstract}
The plethysmo time interval (PTI) is the time interval between the beginning of QRS complex on ECG and the upstroke of the pulse wave on the plethysmogram as measured by a pulse oximeter. We examined whether measuring the PTI has clinical value for evaluating neonatal peripheral circulation. We correlated PTI values and measured, from the hand and foot, height and body weight of 14 neonates. PTI was strongly correlated with height $\left(R^{2}=0.85\right)$ and body weight $\left(R^{2}=0.78\right)$. Height was especially highly correlated, because PTI is principally affected by the distance from the heart to the measured site. We also measured PTI on three clinical cases (patent ductus arteriosus, hypovolemia, and persistent pulmonary hypertension of the newborn). PTI was shortened in cases of peripheral circulatory impairments, because pulse wave velocities increased due to the contraction of arterioles. We conclude that the PTI can evaluate the peripheral circulatory status of the neonate by applying a new principle of pulse oximeter that is widely used in neonatal intensive care units. (Pediatr Res 33: 653-657, 1993)
\end{abstract}

\section{Abbreviations}

PTI, plethysmo time interval

PDA, patent ductus arteriosus

PPHN, persistent pulmonary hypertension of the newborn EMI, electric mechanical interval

In current neonatal intensive care units, very tiny infants who have very fragile skin are covered with many monitoring devices. We are attempting to minimize the number of monitors by expanding functions of the most commonly used monitoring device, the pulse oximeter, to detect changes of peripheral circulation on the neonate. This effort is prompted by our belief that changes in peripheral circulation are an extremely important sign of first manifestation of deterioration of the neonate.

If we could evaluate peripheral circulation objectively, we could manage many diseases at an earlier stage than current devices could detect. Currently, however, no devices are available to monitor peripheral circulation of the neonate noninvasively and continuously.

The plethysmography of the pulse oximeter expresses changes in arteriolar blood volume $(1,2)$. Moreover, the pulse wave velocity increases concurrently with reflex contraction of arterioles (3). Therefore, we examined whether the PTI, the pulse wave conduction time, has clinical value for evaluating the neonatal peripheral circulation.

\section{MATERIALS AND METHODS}

Study 1: relation between $P T I$ and height and body weight. We evaluated the correlation of PTI, measured from hands and feet with height and body weight, of neonates. Fourteen neonates, born at 23 to $39 \mathrm{wk}$ of gestation, and weighing 523 to $2925 \mathrm{~g}$ at birth, were enrolled into the study. PTI are measured in a stable state (mean, d 20). Postconceptional ages at the time of measurement were 25 to $39 \mathrm{wk}$.

Study 2: clinical cases. We examined whether change in PTI have clinical relevance by evaluating peripheral circulation on several clinical cases.

We measured PTI on three clinical cases (PDA, hypovolemia, and PPHN) before and after clinical improvement.

Method. By using a pulse oximeter (Nellcor N-200) and an electrocardiogram monitor, ECG and pulse wave plethysmogram were recorded synchronously by a MacLab system (ADI, Ltd., Castle Hill, Australia). The PTI was measured on a composite graph of ECG and the plethysmogram from the pulse oximeter as shown in Figure 1. PTI was assessed as the time interval between the beginning of QRS complex on ECG and the upstroke of pulse wave on plethysmogram from the pulse oximeter. The time constant, which represents minimal electronic delay in the processing of stored data to display pulse wave form, was in a range of $30 \pm 10 \mathrm{~ms}$.

Differences of means were tested for statistical significance with unpaired $t$ tests. Statistical significance was set at a $p$ value less than 0.05 .

\section{RESULTS}

Study 1. Figure 2 shows the correlations of upper- and lowerlimb PTI with height at the time of measurement $(r=0.85$ and 0.79 , respectively). Figure 3 shows the correlations of upper- and lower-limb PTI with body weight at the time of measurement. The correlations of PTI with body weight were lower than those of height.

Although the number of cases we studied was limited, the PTI were highly reproducible. Moreover, PTI were highly correlated with height and body weight of neonates. Correlations between

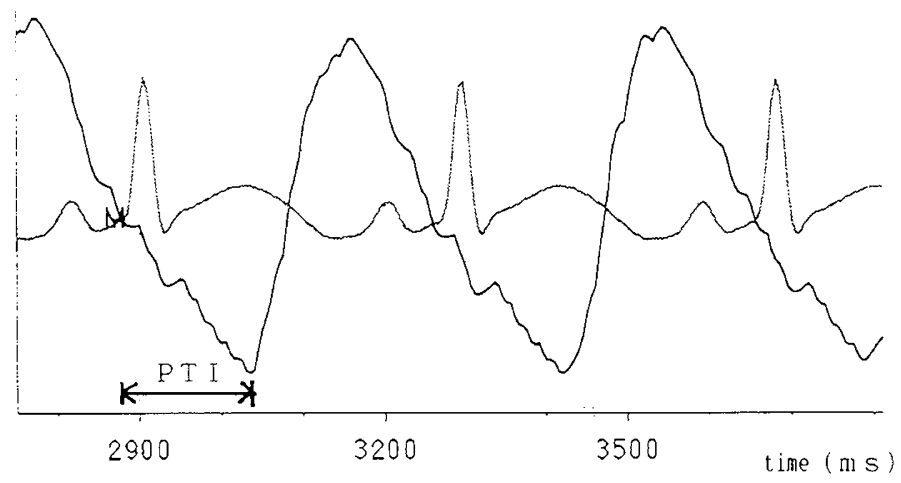

Fig. 1. The PTI is defined as the time interval between the beginning of the QRS complex on the ECG and the upstroke of the pulse wave on the plethysmogram. 


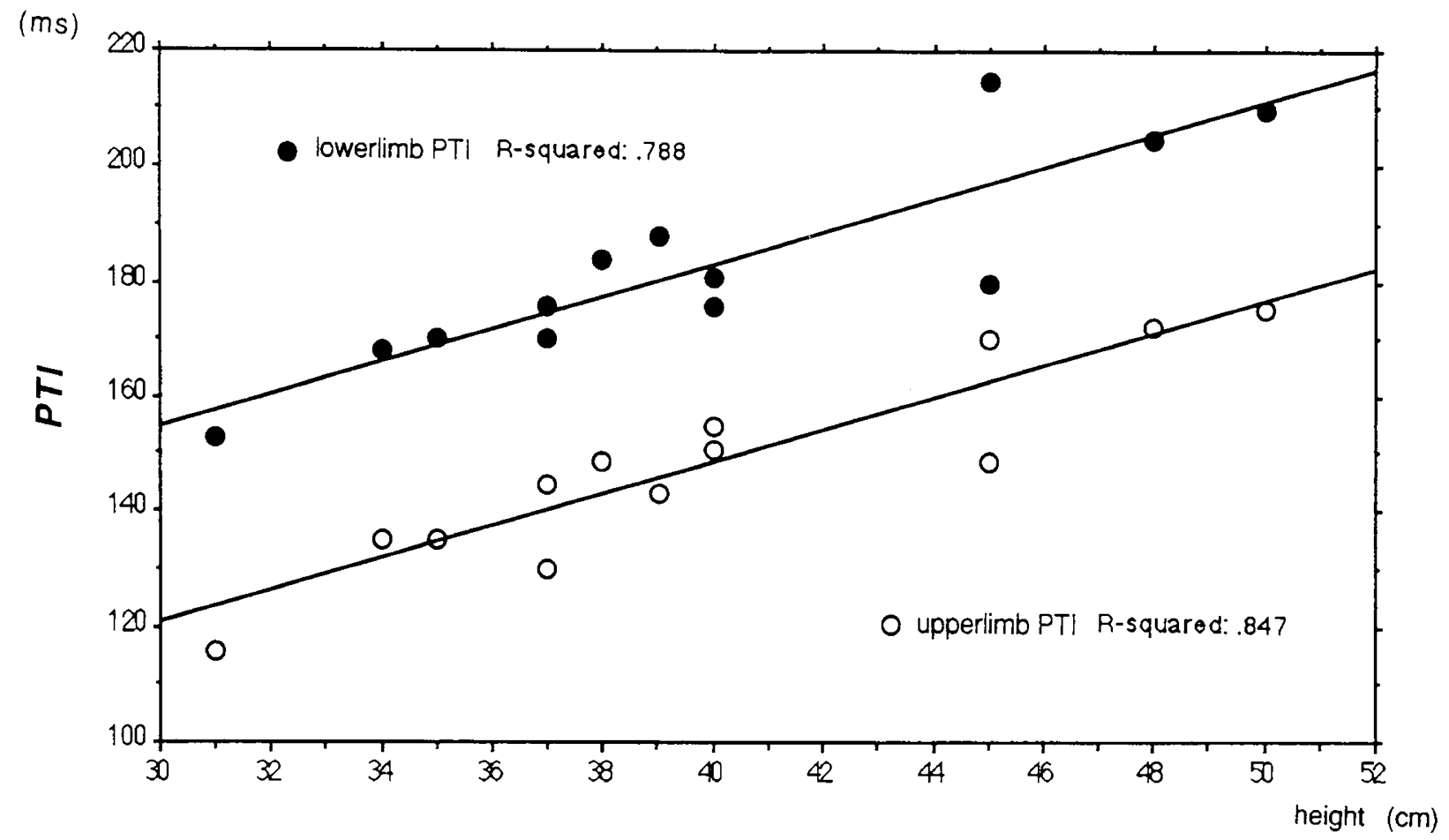

Fig. 2. The correlation of upper-limb $(O)$ and lower-limb PTI $(\bullet)$ with height.

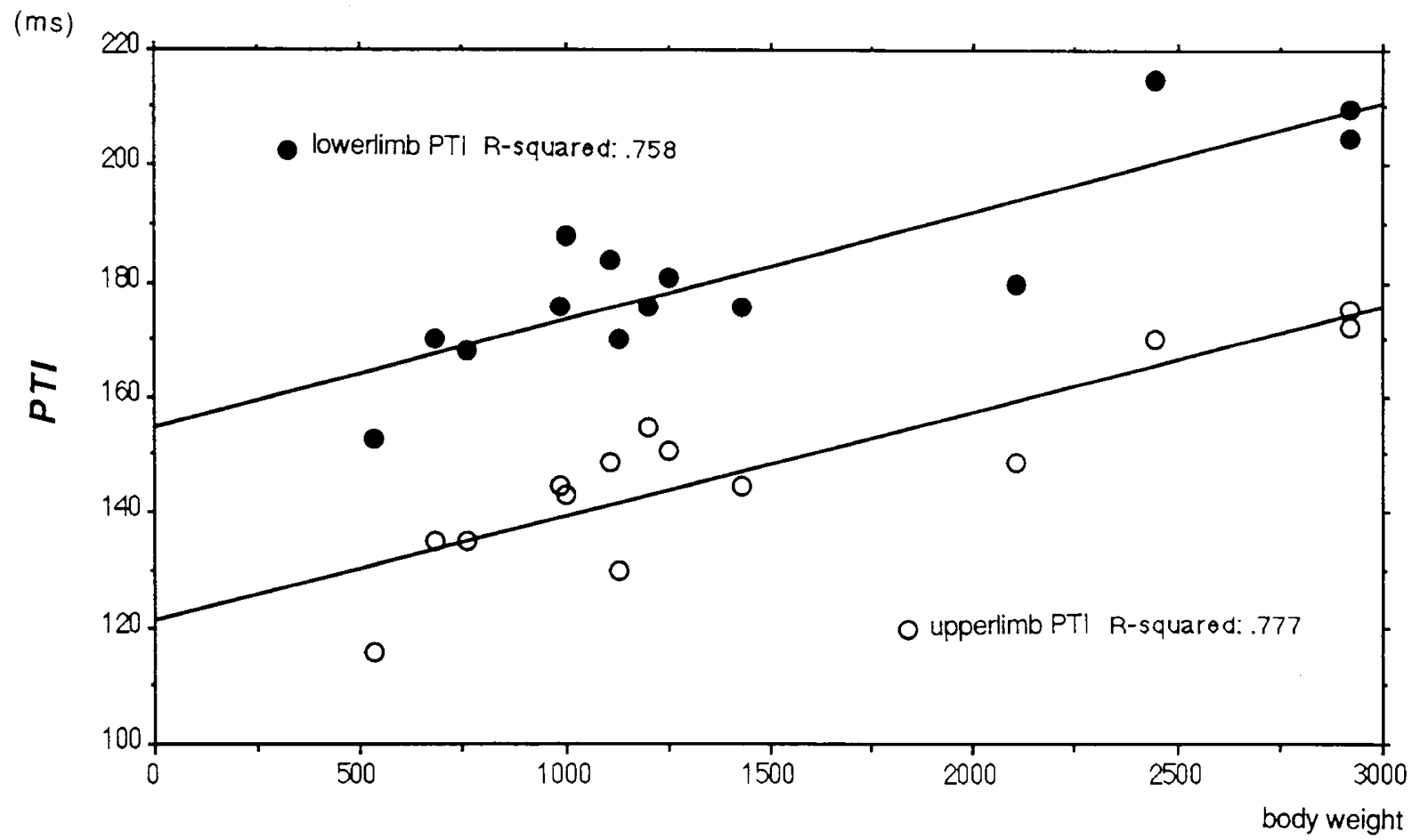

(g)

Fig. 3. The correlation of upper-limb $(O)$ and lower-limb PTI $(\bullet)$ with body weight.

PTI and height were especially high, because PTI is mainly affected by the distance from the heart to the measured site.

Study 2. Case 1: PDA. Figure 4 shows the change of PTI on a case of PDA. We compared PTI changes before and after using an antiprostaglandin synthetic drug to close PDA. The PTI for lower- and upper-limb measurement were 168 and $135 \mathrm{~ms}$, respectively, at $15 \mathrm{~d}$ of age under stable conditions without PDA. However, when PDA became symptomatic, PTI values were shorter than those on normal conditions. PTI became prolonged along with the clinical improvement after administration of an antiprostaglandin drug to close PDA pharmacologically. No significant blood pressure change was observed during the study.

Case 2: hypovolemia. Figure 5 illustrates PTI changes during hypovolemic status of a neonate who was treated by blood exchange transfusion because of $\mathrm{Rh}$ incompatibility. During the procedure, excess blood was accidentally withdrawn, which resulted in a hypovolemic status on the patient. The upper-limb PTI at the preexchange transfusion was $174 \mathrm{~ms}$. During the hypovolemic condition, PTI became shortened significantly, indicating peripheral vasoconstriction induced by the hypo- 


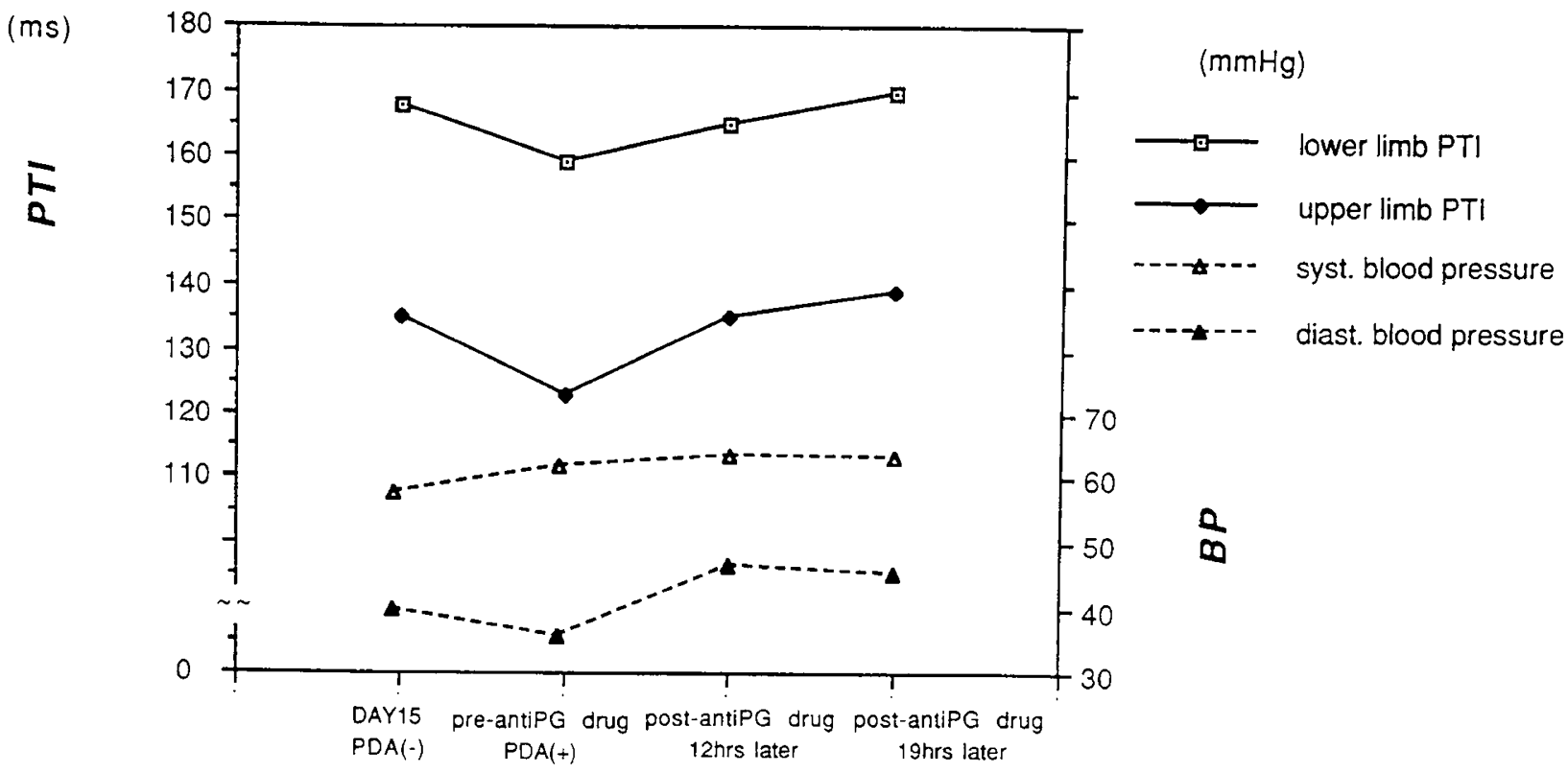

Fig. 4. Change of PTI in a neonate with PDA. $B P$, blood pressure.

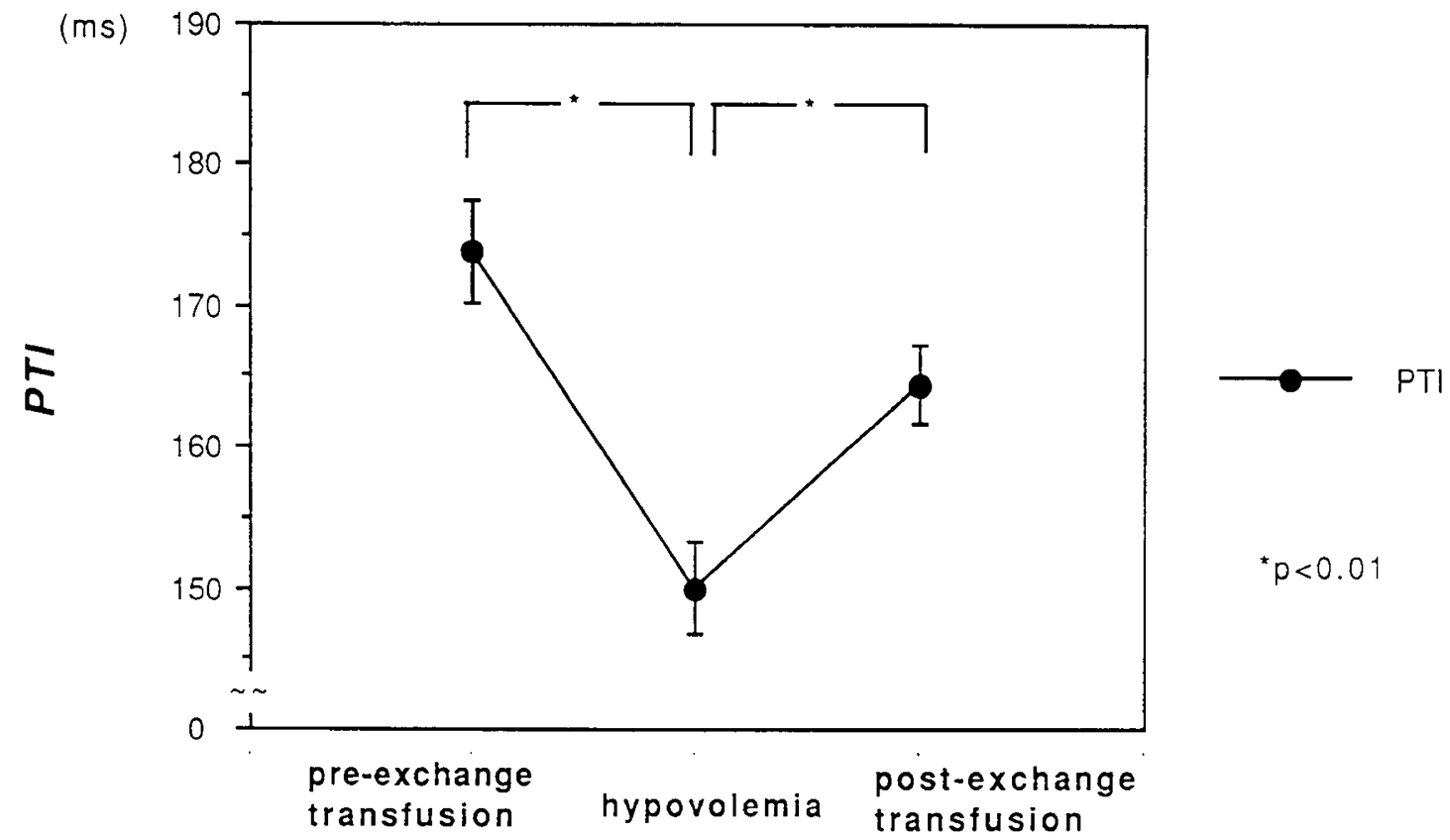

Fig. 5. Change of PTI at hypovolemia.

tension. PTI values normalized after restoring blood volume by transfusion.

During these observations, blood pressure changes were not significant, indicating their PTI changes were independent of blood pressure variations.

Case 3: PPHN. Figure 6 shows PTI values from a case of PPHN due to meconium aspiration syndrome and asphyxia. After using the prostaglandin E1, the upper-limb PTI became prolonged, together with improvement of the infant's general condition. However, at $38 \mathrm{~h}$ after birth, when the patient again deteriorated, PTI became shorter. After administration of trazoline, PTI again became prolonged as the clinical status improved. EMI, which represent the time interval between the beginning of QRS complex on ECG and upstroke of arterial blood pressure, were also measured but showed no significant changes. These latter findings illustrate that PTI change reflects the clinical condition more sensitively than EMI and blood pressure changes.

\section{DISCUSSION}

Investigation of pulse wave characteristics has focused principally on issues related to basic physiology. This study focuses on pulse wave characteristics related to clinical issues. In the 1930s, Kramer and Schulze (4), Matthes (5), and Hertzman (6) developed photoplethysmography. Since that time, the device has been used in clinical fields (7-9). Pulse wave analysis is available with peripheral circulatory impairment and for intraoperative monitoring of circulatory function (10). In the neonatal field, volume pulse wave is used as the basic principle of pulse oximeter (1).

The pressure pulse wave is the transmission of pressure pulse produced by the heart beat through the artery. The pulse wave detected by the pulse oximeter, however, is not the pressure pulse wave, but is the volume pulse wave, which is affected by the elasticity and diameter of the artery. Both pulse wave transmission times are shortened by the hardening or contraction of the 


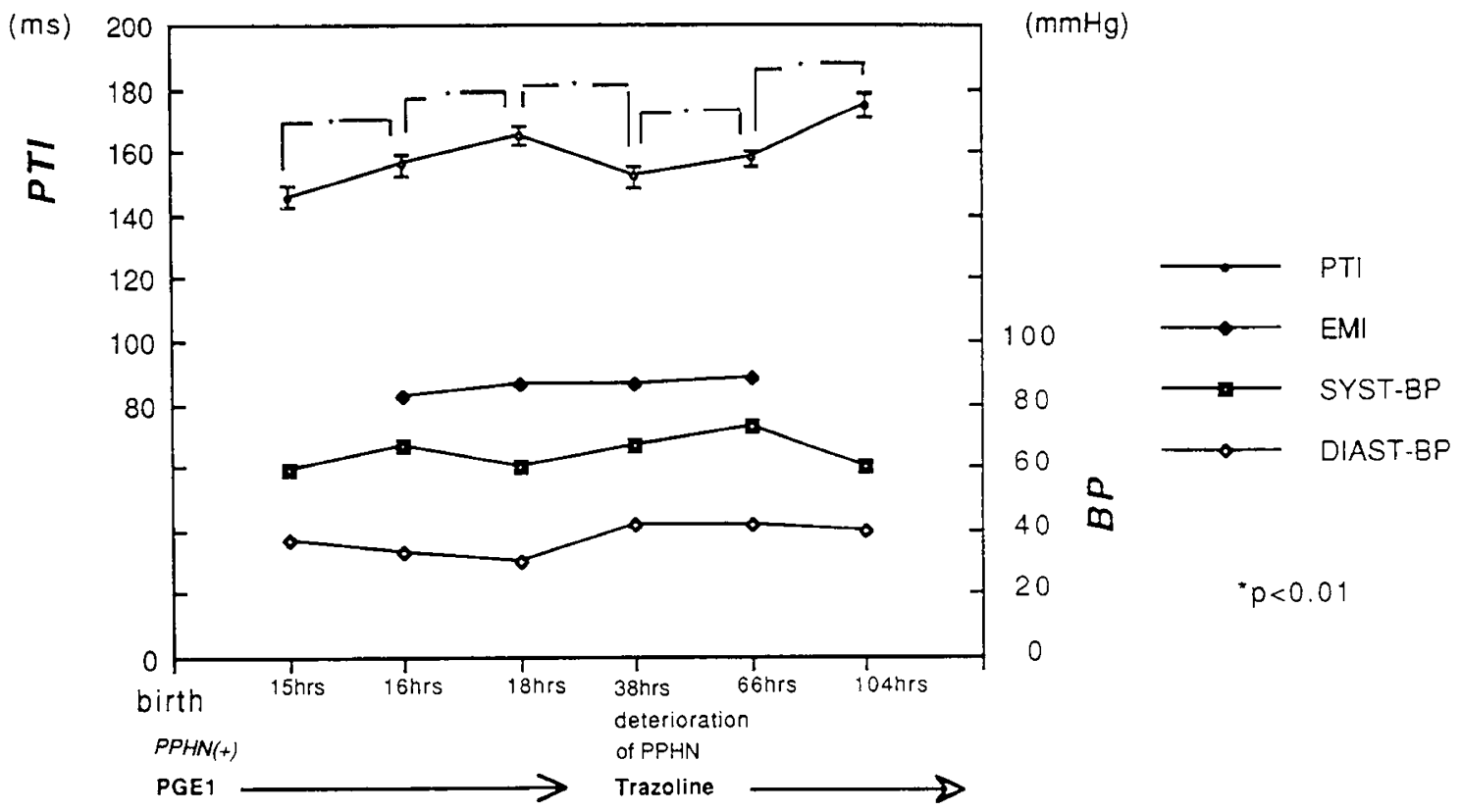

Fig. 6. Change of PTI at PPHN. BP, blood pressure; $P G E 1$, prostaglandin E1.

artery (11-14). In adults, pulse wave transmission times become shorter in cases of arteriosclerosis or hypertension because of reduced elasticity of the arterial wall (15-19). The beginning of pulse wave transmission time is the time of opening of the aortic valve. However, our definition of pulse wave transmission time is the time interval from the beginning of QRS complex on the ECG, which is the onset of electric contraction of the heart to the upstroke of the pulse wave on the plethysmogram from the pulse oximeter.

The influential factors on pulse wave transmission velocity are characteristics of the vessel that are defined principally by wall thickness and elasticity, the inside diameter of the vessel, blood viscosity, and blood pressure, most of which are based on Young's formula (11). However, in case of neonates, vessel wall characteristics on PTI are negligible, because arteriosclerosis is absent. If blood pressure is stable, pulse wave velocity of the neonate is not affected by the compliance of the great arteries, but is chiefly affected by change in diameter of the arterioles. Therefore, PTI changes of the neonate mostly will represent changes of peripheral vascular resistance. Diagrammatic representation of mechanisms of PTI change is illustrated in Figure 7.

PTI also can be affected by cardiac function per se, such as blood pressure change or prolongation of systolic time interval. However, our clinical experiences revealed that PTI changes as the expression of peripheral vasoconstriction were observed before the appearance of parameters of cardiac dysfunction. Therefore, it can be said that PTI change is a useful parameter to

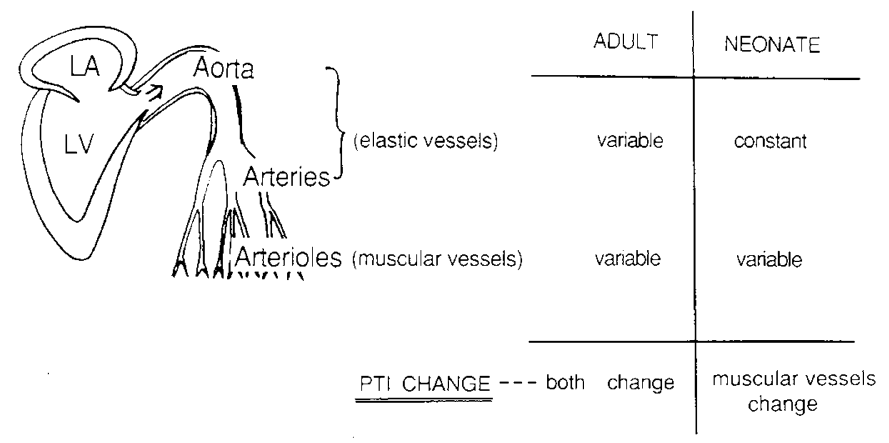

Fig. 7. Mechanisms influencing PTI changes. $L A$, left atrium; $L V$, left ventricle. provide earlier signs of deterioration than systolic time interval and blood pressure changes. Because the pulse oximeter has been widely used as one of the most popular monitoring devices on the neonate, PTI as additional information from the pulse oximeter will help us to assess the clinical condition of the sick neonate.

PTI calculated from pulse wave on pulse oximeter and QRS complex of ECG correlates positively with height and body weight of the neonate. PTI are shortened with peripheral circulatory impairments, which is probably due to the increase in pulse wave velocity resulting from contraction of arterioles. Therefore, PTI can be used as a new denominator to evaluate peripheral circulatory status of the neonate.

Acknowledgments. The authors thank Prof. Gould of the University of California, Berkeley, and Prof. Harper of the University of California, Los Angeles, for their useful advice.

\section{REFERENCES}

1. Payne JP, Severinghaus JW 1986 Pulse Oximetry. Springer-Verlag, Berlin, pp $19-27$

2. Bernstein EF 1985 Noninvasive Diagnostic Techniques in Vascular Disease CV Mosby, St. Louis, pp 97-118

3. McDonald DA 1968 Regional pulse-wave velocity in the arterial tree. J Appl Physiol 24:73-78

4. Kramer K, Schulze W 1948 Die Kaltedilataion der Hautgefa $\beta$-e. Pflugers Arch 250:141-170

5. Matthes K 1961 Kreislauf Untersuchungen am Menschen mit Fortlaufende Registrierenden Methoden. Georg-Thieme, Stuttgart, Germany

6. Hertzman AB 1938 The blood supply of various skin areas as estimated by the photoelectric plethysmograph. Am J Physiol 124:328-340

7. Hawn JA, Rhynear GS, Briston JD 1971 Forearm blood flow and venous tone in acute myocardial infarction. Circulation 44(suppl II):175(abstr)

8. Vyden JK, Nagasawa K, Groseth-Dittrich M, Swan HJC 1975 Peripheral hemodynamics in acute myocardial infarction. Clin Res 23:213 A(abstr)

9. Seino Y, Akaike M, Hayakawa H 1981 Changes of peripheral circulation in patients with acute myocardial infarction: noninvasive observation by the lower limb venous occlusion plethysmography (in Japanese). Kokyu to Junkan 29:297-304

10. Endo M 1987 Clinical study of peripheral vascular changes during anesthesia: evaluation with the venous occlusion plethysmograph (in Japanese). Masui 36:704-712

11. Yoshimura M, Mishima Y 1972 Clinical Features of Pulse Waves (in Japanese) Igaku Shoin Ltd, Tokyo

12. Evans ML, Geddes LA 1988 An assessment of blood vessel vasoactivity using photoplethysmography. Med Instrum 22:29-32

13. Cooke ED, Bowcock SA, Smith AT 1985 Photoplethysmography of the distal pulp in the assessment of the vasospastic hand. Angiology 36:33-40

14. lida N 1992 Dynamic properties of large arteries assessed from finger and toe pulse waves. Myakkangaku 32:503-510 
15. Steele JM 1937 Interpretation of arterial elasticity from measurements of pulse wave velocities. Am Heart J 14:452-465

16. Strandness DE, Gibbon GE, Bell JW 1961 Use of a new simplified plethysmograph in the clinical evaluation of patients with arteriosclerosis obliterans. Surg Gynecol Obstet 112:751-756

17. Schimmler W 1966 Correlations between the pulse wave velocity in the aortic illiac vessel, and age, sex and blood pressure. Angiology 17:314-322
18. Jespensen LT, Krusell LR, Lederballe O 1987 Photoplethysmographic assessment of digital arterial compliance in the diagnosis and treatment of hypertension. J Clin Hypertens 3:368-376

19. Ferrara LA, Strazzullo P, Giumetti D, Annuzzi G, Pasanisi F, Mancini M 1985 Peripheral circulation in hypertensive patients: finding by digital pulse plethysmography. Artery 12:346-351

\section{Erratum}

On page 376A of the April program issue (Pediatric Research, Volume 33, Issue 4, Part 2), abstract 2296 was inadvertently omitted by the printer and abstract 2297 appeared in its place. Abstract 2297 on page 387A is correct as printed, and abstract 2296 should have appeared as follows.

\section{6}

SURFACTANT FUNCTION IN VIVO: INCONGRUITY OF THE SURFACE MONOLAYER THEORY. Emile $\mathrm{M}_{2}$ Scarpelli, and Alan J. Mautone. Perinatology Center, Cornell University Medical School, NY, NY and Depts. of Anesthesiology and Pediatrics, New Jersey Medical School, Newark, NJ

The surface monolayer theory of Clements, et al explains surfactant function in vivo as a process in which surfactant films at the alveolar air-liquid interface are refined to virtually pure DPPC during compression from total lung capacity (TLC) to functional residual capacity (FRC), where stable near zero surface tension $(\gamma)$ is established and sustained during tidal volume $\left(V_{\nu}\right)$ breathing. We tested the theory on normal surfactant extract from calf lung in a leak-proof surface balance that models the open film of the theory. The ausorbed film was cycled between $100 \%$ and $20 \%$ surface area (SA) until $\gamma$-SA isotherms were reproducible. Cycling was continued after decompression to $100 \%$ SA (simulated TLC) with one of the following sequences: [1] Compression to $\approx 80 \%$ SA ( $\approx$ FRC for upper lung with one of the following sequences: [1] Compression to $\approx 80 \% \mathrm{SA}(\approx \mathrm{F}$ ) for upper lung $5 \%$ to $25 \%$ SA changes to simulate a range of $V_{1}$ breathing patterns; $[2]$ compression to middle zone FRC ( $\approx 53 \% \mathrm{SA})$, then $V_{1}$ breathing; and [3] compression to lower zone FRC $(\approx 36 \% \mathrm{SA})$, then $\mathrm{V}$, breathing. Upper zone $\gamma$ was $>10 \mathrm{dyn} / \mathrm{cm}$ at FRC and increased to $>>10 \mathrm{dyn} / \mathrm{cm}$ (minimum $\gamma$ ) and $>30 \mathrm{Jyn} / \mathrm{cm}(\gamma \max )$ during $V_{1}$ cycling. Lower and middle zone $\gamma$ at FRC, $\approx 0$ to $4 \mathrm{dyn} / \mathrm{cm}$, increased in 1 to $3 \mathrm{~V}_{1}$ cycles to $>10 />30 \mathrm{dyn} / \mathrm{cm}$ $(\gamma \min / \gamma \max ) . \gamma$ increased spontaniously from $\approx 0$ to $>10 \mathrm{dyn} / \mathrm{cm}$ in $<2 \mathrm{sec}$ when compression was stopped at FRC (simulated end-expiratory pause from TLC). In vivo, the pressure due to $\gamma$ during $V_{1}$ breathing and at end-expiration would be $\approx 2$ to $>5 \mathrm{~cm} \mathrm{H}_{2} 0$ in adult alveoli, $125 \mu \mathrm{m}$ radius $(\mathrm{r}) ; \approx 8$ to $>24 \mathrm{~cm} \mathrm{H}_{2} \mathrm{O}$ in neonatal alveoli, $25 \mu \mathrm{m} \mathrm{r}$; and $>400 \mathrm{~cm} \mathrm{H} \mathrm{H}_{2} \mathrm{O}$ in alveolar corners, $0.5 \mu \mathrm{m} \mathrm{r}$. Clearly, $\gamma$ and pressure are too high as the monolayer fails to follow the $\gamma$ history predicted by the theory under conditions of neonatal and adult breathing in all regions of the lung. Supported by HL-38303 to AJM. 